\title{
Comparative genomic analysis of moderate bacteriophages of alfalfa root nodule bacteria
}

\author{
Kozlova A.P. \\ All-Russian research institute for \\ agricultural microbiology, Pushkin, \\ Saint-Petersburg, Russia \\ alexsandrak95@mail.ru \\ Antonova E.V. \\ Institute of Plant and Animal Ecology, \\ Ural Division of Russian Academy of \\ Sciences, Ekaterinburg, Russia \\ selena@ipae.uran.ru \\ Dzyubenko E.A. \\ Federal Research Center N. I. Vavilov \\ All-Russian Institute of Plant Genetic \\ Resources (VIR) Ministry of science \\ and higher education, Saint-Petersburg, \\ Russia \\ elena.dzyubenko@gmail.com
}

\author{
Muntyan V.S. \\ All-Russian research institute for \\ agricultural microbiology, Pushkin, \\ Saint-Petersburg, Russia \\ vucovar@yandex.ru
}

Muntyan A.N. All-Russian research institute for agricultural microbiology, Pushkin, Saint-Petersburg, Russia allmuntyan@gmail.com

Roumiantseva M.L. All-Russian research institute for agricultural microbiology, Pushkin, Saint-Petersburg, Russia mroumiantseva@yandex.ru
Afonin A.M.

All-Russian research institute for agricultural microbiology, Pushkin, Saint-Petersburg, Russia afoninalexeym@gmail.com

Kabilov M.R.

ICBFM SB RAS, Novosibirsk, Russia kabilov@niboch.nsc.ru.

\begin{abstract}
AP300 infecting Sinorhizobium meliloti was isolated from soil sample from mountainous region of Dagestan, which belongs to the Caucasus Center for Legume Variety in the frame of the grant RSF 17-1601095. The AP300 genome was annotated and its similarity to bacteriophage vB MloP_Lo5R7ANS (NC_025431; Podoviridae family) infecting Mesorhizobium loti strains was revealed. In the chromosomes of 4 strains of Sinorhizobium meliloti isolated in geographically different regions from soils and nodules of alfalfa plants, intact vB MloP_Lo5R7ANS-like prophages were detected. The $46.39-57.14 \%$ ORFs of AP300 and 4 prophages were homologous to ORFs of $\mathrm{VB}$ MloP_Lo5R7ANS. The 18 ORFs from mentioned above ORFs were identified in all studied sequences and they were similar to those in vB MloP L05R7ANS. The other 7.22-15.79\% ORFs were homologous to other phages. ORFs encoding virion components: head, capsid, tail, virion, integrase were detected in soil bacteriophage AP300 and in all studied prophages determined in rhizobia symbionts of alfalfa.
\end{abstract}

Keywords - bacteriophage, prophage, root nodule bacteria, tail tubular protein A, comparative genomic research.

Motivation and aim

\section{Motivation}

Bacteriophages are genetically diverse biological objects which are abandoned on the Earth, occurring on average $1 *$ $10^{31}$ particles [1]. Bacteriophages can cause lytic infection, accompanied by reprogramming of the host metabolism, destruction of the host cell, or can integrate into the bacterial chromosome in the result of lysogenic infection and transmitted to future generations [2, 3]. It was shown that phages can significantly reduce the number of strains in soil microbiomes as well as in rhizosphere of legume host plant, and affect on the cultural or symbiotic properties of rhizobia [4].

The specificity of phage infection toward bacterial cell is related to tail proteins of the bacteriophage. For example, in vB MloP_Lo5R7ANS the three types of tail proteins involved in the bacterial infection mechanism were annotated. They are tail tubular A protein (TTPA), tail tubular B protein (TTPB), and tail fiber protein (TFP). The latest protein is responsible for recognizing host membrane receptors and for initiating the infection process. The TTPA is a structural protein that is located in the tail of the bacteriophage. It plays an important role in phage adhesion on bacterial cell wall, which is a prerequisite for host infection. For TTP A and B, depolymerization activity in relation to exopolysaccharides of bacteria capsule was shown [6].

For Sinorizobium bacteriophages or prophages comparative genomic research and annotation of common specific proteins have not been carried out before.

\section{Aim}

The aim of the study was a comparative genomic research of prophages identified in native isolates and phages recovered from soil in order to search common sequences related phage adhesion and infection.

\section{Methods}

A pure culture of the bacteriophage Sinorhizobium meliloti AP300 was obtained by the Adam's double-layer agar method from a phagolysate obtained from the soils of the mountainous region of Dagestan by $S$. meliloti L5-30 trap strain.

The genomes of the AT7-2 and Ur106 strains and the AP300 bacteriophage were sequenced by short reading method (MiSeq, Illumina, USA). Complete genomes of S. meliloti strains were sequenced by Nanopore sequencing (Oxford Nanopore Technologies, UK). Assembling to circular replicons and annotating genomes was performed by bioinformatics approaches (SPAdes, denovo assembler Flye, Racon and Medaka modules, Pilon software tool, Prokka annotation software package for bacteria). Genome sequences of strains of $S$. meliloti RU11 / 001 and AK21 were obtained by GenBank (NZ CP021219.1 and NZ CP026525.1). Prophages were searched on the chromosomes of $S$. meliloti strains by Phaster web-server; comparative analysis was performed by BLASTn, BLASTp, Prosite, I-TASSER, SWISS-MODEL, PDBflex resources. 


\section{Results}

The four prophages were identified in genomes of $S$. meliloti strains isolated from different geographical areas, and genome of bacteriophage isolated using alfalfa nodule bacteria were screened and sequences homologous to the bacteriophage vB MloP Lo5R7ANS Mesorhizobium loti, were revealed.

The corresponding sequences identified in AP300 phage was shorter $(42.8 \mathrm{~kb})$ then genome size of $\mathrm{vB}$ MloP Lo5R7ANS (45.7 kb), while homologous sequences in prophages were ranged from 44.1 to $60.2 \mathrm{~kb}$.

The GC content was: $61.02 \%$ in AP300 and in prophages it was ranged from 60.65 to $61.13 \%$, in comparison with $61.05 \%$ of $\mathrm{vB}$ MloP Lo5R7ANS. The number of ORFs was 60 for AP-300, while vB MloP Lo5R7ANS contained 64. In cases of prophages the number of ORFs was varied from 55 to 97. The AP-300 and $\mathrm{vB}$ MloP Lo5R7ANS genomes carried leucine tRNA, and the sequences of prophages were inserted into leucine tRNA in the chromosomes of the strains. The 46.39-57.14\% ORFs of AP300 and prophages were homologous to ORFs of vB MloP Lo5R7ANS (Evalue $<1 *$ $\left.10^{-10}\right)$. The 18 ORFs were identified in all studied sequences and similar to those of vB MloP Lo5R7ANS. The other 7.22$15.79 \%$ ORFs of bacteriophage and prophages were homologous to other phages $\left(\mathrm{E}_{\mathrm{value}}<1 * 10^{-14}\right)$. For the AP300 bacteriophage and three prophages, 19-22 unique ORFs were detected. The prophage of strain Ur106 had 45 unique ORFss, due to the copy number of 28 ORFs (from 2 to 5), which amounted to $46.39 \%$ of the ORFs of its entire sequence. In studied sequences were found ORFs encoding virion components: head, capsid, tail, virion, integrase, which were similar to a corresponding set of ORFs in $\mathrm{vB}$ MloP Lo5R7ANS. In AP300 as well as in sequences of prophages the enzyme endolysin were annotated, and in case of prophage of Ur106 the transposase TRm23a was identified.

The analysis of amino acid sequences provided the next information that the number of ORFs encoding proteins with known functions was varied from 6 to 8 ORFs in studied sequences.

In each phage related sequences the tail tubular protein $\mathrm{A}$ (TTPA) was annotated. A comparative analysis of the amino acid sequences of TTPA of moderate bacteriophages and $\mathrm{vB}$ MloP_Lo5R7ANS was done in silica. The size of TTPA was 193 a.o. in prophages, and it was 195 a.a. in vB MloP Lo5R7ANS. The level of homology was $57.29 \%$ (for AP300, and prophages of strains RU11/001, Ur106) (I = $57.29 \%, \mathrm{Cov}=98 \%$, Evalue $=4 * 10^{-78}$ and $57.81-58.33 \%$ for prophages of strains AT7-2 and AK21). The amino acid sequences of the TTPA proteins of the prophages and AP-300 lost two amino acid residues: asparagine and serine and had up to 88 amino acid substitutions compared to vB MloP_Lo5R7ANS.

In total $12-13$ different protein modification sites were established for studied sequences. They are: one protein glycosylation site in TTPA, 4 protein kinase C phosphorylation sites, and 5 type II casein kinase phosphorylation sites. It was revealed that TTPA proteins identified in prophages can have additional one phosphorylation site of protein kinase $\mathrm{C}$ or a site of modification with myristic acid, or in contract could lose up to two phosphorylation sites of casein kinase II and one site of PKC phosphorylation.

Analysis of the tertiary structure of TTPA proteins vB MloP_Lo5R7ANS and phage related sequences in rhizobia strains and in AP300 showed that the closest structural homologue is protein A of the tail tubule of the bacteriophage KP32 of Klebsiella pneumonia (pdb5MU4) (I = 34-37\%, Cov $=94-95 \%$ ), similarity models 5MU4 and TTPA vB MloP Lo5R7ANS was $54.44 \%$, while in case of prophages it was higher and amounted to 56.35 , but in prophage of soil strain AT7-2 and in bacteriophage AP300 it was 58.19\%. Thus, we have determine the presence of sequences the 18 ORFs related to $\mathrm{vB}$ MloP_Lo5R7ANS in sequences of prophages of all studied strains as well as in temperate bacteriophage AP300, while the phage-specific parts in were $33.3-46.39 \%$. The prophage of strain Ur106 has up to 5 copies of one third of the ORFs of its genome. Tail tubular protein A in all studied sequences has structural differences from the currently known TTPA bacteriophage KP32 Klebsiella pneumoniae (pdb5MU4), it has specific sites of posttranslational modification, which can probably determine the specificity of interaction with EPS of nodule bacteria of alfalfa.

\section{Acknowledgment}

Supported by the RFBR 18-04-01278a (analysis of ORF and amino acid sequences) and RSF 17-16-01095 (genome sequencing).

\section{References}

[1] Clokie MR, Millard AD, Letarov AV, Heaphy S. Phages in nature. Bacteriophage. 2011 Jan; 1(1):31-45.

[2] Fortier LC, Sekulovic O. Importance of prophages to evolution and virulence of bacterial pathogens. Virulence. 2013 Jul 1; 4(5):354-65.

[3] Krupovic M, Prangishvili D, Hendrix RW, Bamford DH Genomics of bacterial and archaeal viruses: dynamics within the prokaryotic virosphere. Microbiol Mol Biol Rev. 2011 Dec; 75(4):610-35

[4] Dziewit L., Oscik K., Bartosik D., Radlinska M. Molecular characterization of a novel temperate Sinorhizobium bacteriophage, ФLM21, encoding DNA methyltransferase with CcrM-like specificity. J Virol. 2014 Nov; 88(22): 13111-13124.

[5] Veesler D, Cambillau C. A common evolutionary origin for tailedbacteriophage functional modules and bacterial machineries. Microbiol Mol Biol Rev. 2011 Sep; 75(3):423-33, first page of table of contents.

[6] Pyra A., Brzozowska E., Pawlik K., Gamian A., Dauter M., Dauter Z. Tail tubular protein A: a dual-function tail protein of Klebsiella pneumoniae bacteriophage KP32. Sci Rep. 2017; 7: 2223. Published online 2017 May 22. 